\title{
Photochemistry on a polarisable semi-conductor: what do we understand today?
}

\author{
Divya Tiwari $\cdot$ Steve Dunn
}

Received: 28 February 2009/Accepted: 6 April 2009/Published online: 22 April 2009

(c) The Author(s) 2009. This article is published with open access at Springerlink.com

\begin{abstract}
The continued development of ferroelectric materials into more and more consumer led applications has been at the forefront of recent ferroelectric material research. It is, however, possible to view a ferroelectric as a wide band gap semi-conductor that can sustain a surface charge density. This charge density arises from the movement of ions in the crystal lattice and the need to compensate for this charge. When viewing ferroelectrics as polarisable semi-conductors a large number of new interactions are possible. One such is the use of super band gap illumination to generate electron-hole pairs. These photogenerated carriers can then perform local electrochemistry. What is most interesting for ferroelectric materials is that the REDOX chemistry can be chosen by selectively modifying the domain structure of the ferroelectric-we can perform oxidation and reduction on the surface of the same material at spatially separate locations, or use the material to drive photoexcited carriers apart. This means we can separate the REDOX products or produce patterns of photogenerated material in places we have predetermined. This review aims to introduce the background research that has led to the current understanding as well a highlight some of the current areas that require further development.
\end{abstract}

\section{Introduction}

The review is intended to contextualise the current understanding of the interactions of the surface of a ferroelectric

D. Tiwari · S. Dunn $(\bowtie)$

Nanotechnology Centre, Cranfield University,

Cranfield MK43 0AL, UK

e-mail: s.c.dunn@cranfield.ac.uk under non-equilibrium conditions in the light of excellent work undertaken last century. It shows where the new understanding is knitting together a number of diverse research topics into one thread of understanding. This current work is not intended to give a deep expose on any one topic rather highlight some of the seminal works in topical areas and bind them together. Over the past 5 years there has been an increase in the interest in the use of ferroelectric materials as photocatalysts, or their use in photochemistry. This is due to the ability of a ferroelectric material to be patterned at length scales that range from tens of nanometres to many centimetres. The different regions that have been patterned on the ferroelectric surface are both stable (with only a few exceptions) and exhibit different REDOX chemistry that is determined by the polarisation of the ferroelectric. The possibility of using such materials in a host of optoelectronic devices is also covered due to the effective electron-hole separation that can be achieved.

This review will cover the historical perspective of the semi-conducting nature of ferroelectric materials which is the natural pre-cursor to investigating the photochemistry, and then focus on the new developments of patterned surfaces being used for selected chemical reactions. It will conclude by looking at some of the possible future implications, and applications of the results. Work focussing on the semi-conductor nature of a ferroelectric has shed light on the properties and phenomena occurring on the surface of the ferroelectric material under the non-equilibrium conditions of irradiation by super band gap irradiation. It also gives some indications as to what is happening at the interface between a poled ferroelectric material and its wider environment. Prior to the publication by Kalinin et al. in 2002 [1], where a novel paradigm for the assembly of 3-D nanoparticles on a ferroelectric surface, was 
demonstrated there had been some investigations detailing the photo induced interactions of ferroelectric surfaces and the wider environment. Most of the recent research has been focussing on the nature and reasons behind the preferential growth of nanoparticles on domain-patterned ferroelectric materials. The developed nanoparticles, and nanoparticle patterns, have huge application in the field of bio-medicine, optoelectronic devices, photonics, product labelling and authentication.

Strong electromechanical coupling, a high dielectric constant and the unique property of sustaining spontaneous polarisation below the Curie temperature make ferroelectrics extremely fascinating materials and constitute the basis of wide technological applicability [2-6]. It is true to say that in one form or another ferroelectric materials have become ubiquitous for the society of today. After the discovery of ferroelectricity in Rochelle Salt by Valasek [7] in 1920, ferroelectric materials have emerged as a very promising material group with wide applications such as sensors, actuators, transducers, electronic and electroptical materials and numerous novel applications including microelectromechanical systems [8-10]. Applications range from the use of $\mathrm{BaTiO}_{3}$ in capacitors required for almost every electronic device to improvement of diesel engine performance through accurate control of injection performance. On a more research focused level a new and novel application of ferroelectrics is to facilitate the growth of self-assembling 3D nanostructures.

It is, however, interesting to note that ferroelectric materials were among some of the first materials to be shown to have photoelectric or photochemical properties. The studies on ferroelectric materials followed on from the early work that resulted in the observation that paint containing particles of $\mathrm{TiO}_{2}$ blistered or discoloured under sunlight. It was in 1938 that the first publication showed that $\mathrm{TiO}_{2}$ was photoactive [11]. This was then followed by interesting work that resulted in the Mashio et al. publishing a paper which showed the photoxidation performance of $\mathrm{TiO}_{2}$ [12]. The powerful oxidation performance of the photogenerated hole was highlighted in this work and led to the significant effort to develop the use of $\mathrm{TiO}_{2}$ in photocatalytic and photochemical systems. During the oil crisis in the late 1960s and early 1970s a number of groups picked up on the photostability of semi-conductors as a possible means of generating useful energy, either in the form of an electric current or chemical energy. The result of this work was the Nature paper published in 1972 by Fujishama and Honda [13] which showed the use of semi-conductors in photogenerated cells. It was only a matter of a few years later when the photochemistry of $\mathrm{BaTiO}_{3}$ was reported in a paper by Nasby and Quinn [14], who showed a pH dependence on flat-band potential and that $\mathrm{BaTiO}_{3}$ shows excellent photostability and good photocurrents. This seminal paper left a number of unanswered questions that were a result of the ferroelectric nature of $\mathrm{BaTiO}_{3}$ and the influence that has on the surface photochemistry due to selective movement of photoinduced carriers. The interest in the surface photochemistry of ferroelectrics was largely left untouched for a number of years until around 2000 when Giocondi and Rohrer [15] published a paper highlighting the variation in surface photochemistry for $\mathrm{BaTiO}_{3}$. This paper related the variation in the band bending associated with the domain polarisation of the ferroelectric, this was the first time such a relationship was established for surface photochemistry. It should be noted that although there was little work investigating the surface photochemistry of semi-conductors there was substantial work developing theories to relate the semi-conductor properties of ferroelectric materials [16].

Synthesis of devices with micro and nanoscale dimensions is a focus of intense scientific and technological interest. This is highlighted by the boom in nanotechnology and microtechnology research from all corners of the world. In the UK alone there is now a dedicated Microsystems and Nanotechnology network and associated ring fenced funding.

A variety of interesting approaches have been developed for the growth of nanostructures. Some of the initial nanolithography's were based on scanning probe systems [17] and Microcontact printing [18] which has become a common method of patterning self assembled monolayers and enabled nanoscopic resolution and patterning for a large variety of applications that include nanoelectronics and single molecule transistors. Nanoimprint lithography [19], was developed to pattern semiconductor photo resists, DNA and protein directed assembly for the control of nanoparticle assembly [20-22] have also proven suitable for producing patterns at nanoscale resolutions. Dip-pen nanolithography and approaches based on ink jet printer strategies are being developed and used in applications such as array chips [23, 24]. Finally electric, magnetic or mechanical fields were used to align nanoparticles [25]. During this period of intense research interest Kalinin et al. [1] proposed a novel idea for the assembly of nanostructures, ferroelectric nanolithography, which facilitates the growth of 3D nanostructures on predefined locations on a ferroelectric surface, as shown in Fig. 1. The basic process behind this selective surface chemistry is band bending induced by the internal field of the ferroelectric material, as shown in Fig. 2. Kalinin's work is further supported by Dunn et al. [26] who have shown that it is possible to selectively absorb biological molecules onto the surface of a ferroelectric. A growing theme of work is now to investigate the influence of surface polarisation on chemical sorption $[27,28]$ and physical sorption on the ferroelectric surface [29]. 
Fig. 1 PFM image of lines patterned with alternating +10 and $-10 V_{\mathrm{dc}}(\mathbf{a})$. Bright areas are positive domains and dark areas are negative domains. An image of the surface topography (b) after deposition of $\mathrm{Ag}$ nanoparticles. Note that the metal has deposited only on the positive domains. PFM image of checkerboard domain structure (c) and SEM image of corresponding silver photodeposition pattern (d) (with permission from Ref. 1)
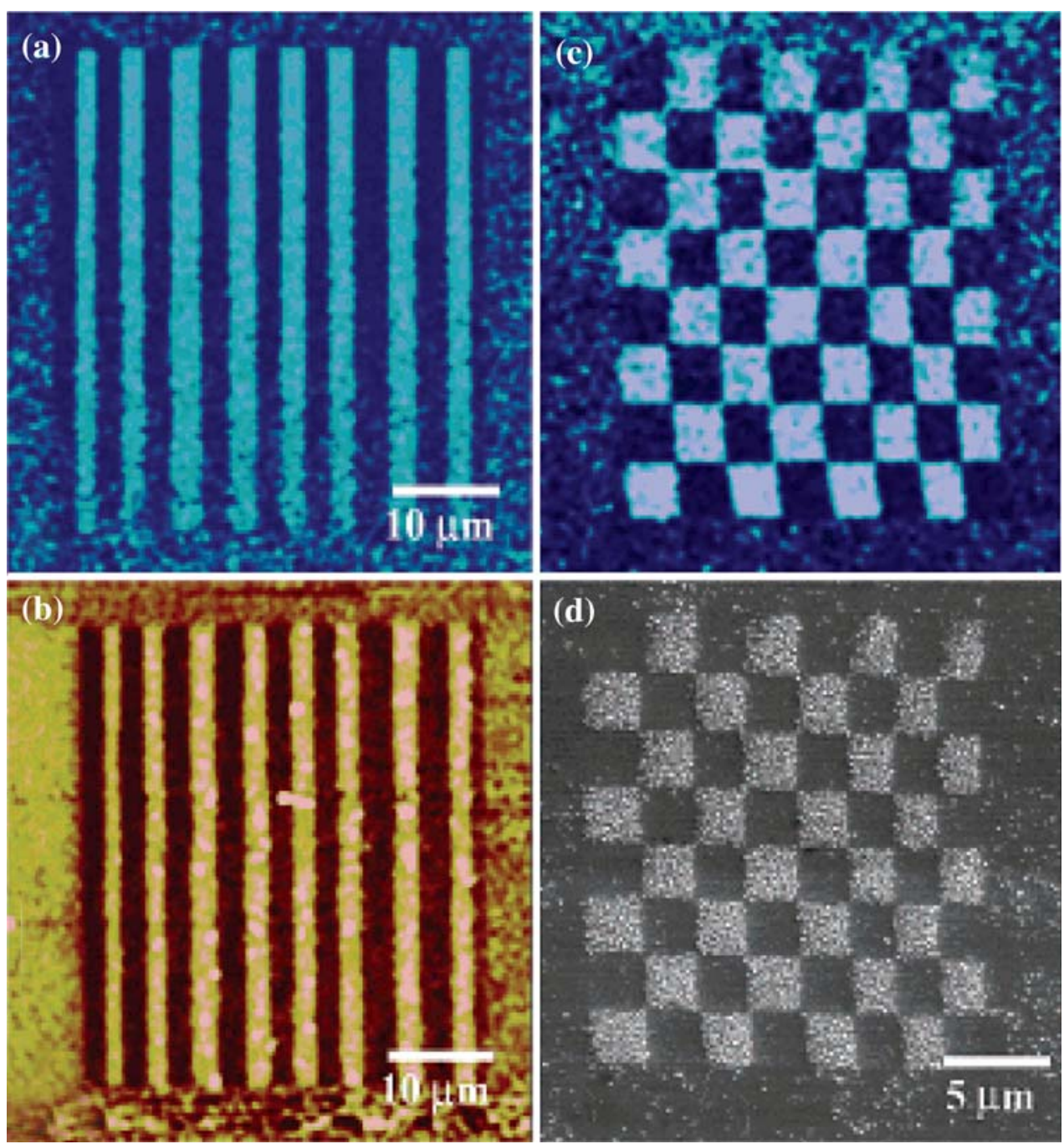

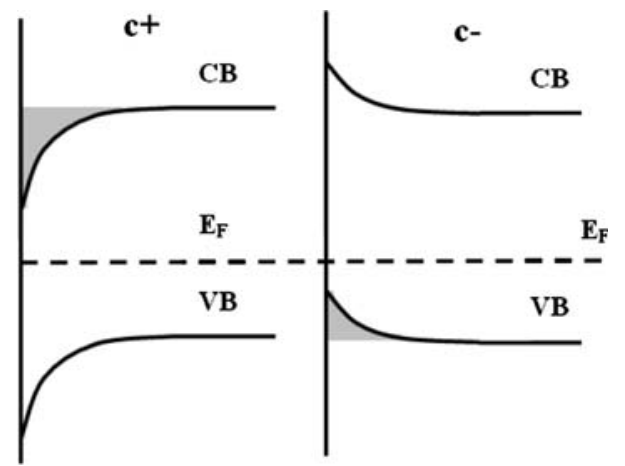

Fig. 2 Configuration of the band edges at the surface of polarised ferroelectric. In $\mathrm{c}^{+}$domains (left), the depolarisation field causes downward band bending and $\mathrm{h}^{+}$depletion. Photogenerated $\mathrm{e}^{-}$will move towards the surface and can take part in reduction reactions. In $\mathrm{c}^{-}$domains (right), the bands are bent upwards and there is $\mathrm{h}^{+}$ accumulation at the surface. Photogenerated $\mathrm{e}^{-}$will move away from the surface promoting oxidation reactions by the presence of accumulated and photogenerated $\mathrm{h}^{+}$(with permission from Ref. 35)
The development of ferroelectric nanolithography can be considered as a combination of two phenomena:

1 The property of atomic polarisation to control local domain structure in ferroelectric material, through patterning with piezo force microscopy (PFM) [30, 31] or electron beam systems [32].

2 Using the photo-oxidation/photoreduction reactions to direct the assembly of nanoparticles on selected regions of the ferroelectric surface.

What is now being commonly accepted is that the chemical reactivity of a ferroelectric surface is dependent on the polarisation of the domain that is underlying the surface [33]. This was successfully demonstrated by Giocondi and Rohrer by the growth of Ag particles on ferroelectric surface through the process of photoreduction [15]. In this case the reduction of silver cations to silver metal was found to occur on the positive domains on $\mathrm{BaTiO}_{3}$, see 
Fig. 3 Spatial separation of REDOX products for photochemical reduction of silver nitrate on the surface of $\mathrm{BaTiO}_{3}$, a before reaction giving an indication of domain patterns in the substrate material and $\mathbf{b}$ after illumination under silver nitrate where only one type of domain has been covered in silver, as indicated by the bright white contrast. A high resolution image before (c) and after (d) illumination showing the selective deposition of silver on the surface of the $\mathrm{BaTiO}_{3}$ (with permission from Ref. 15)
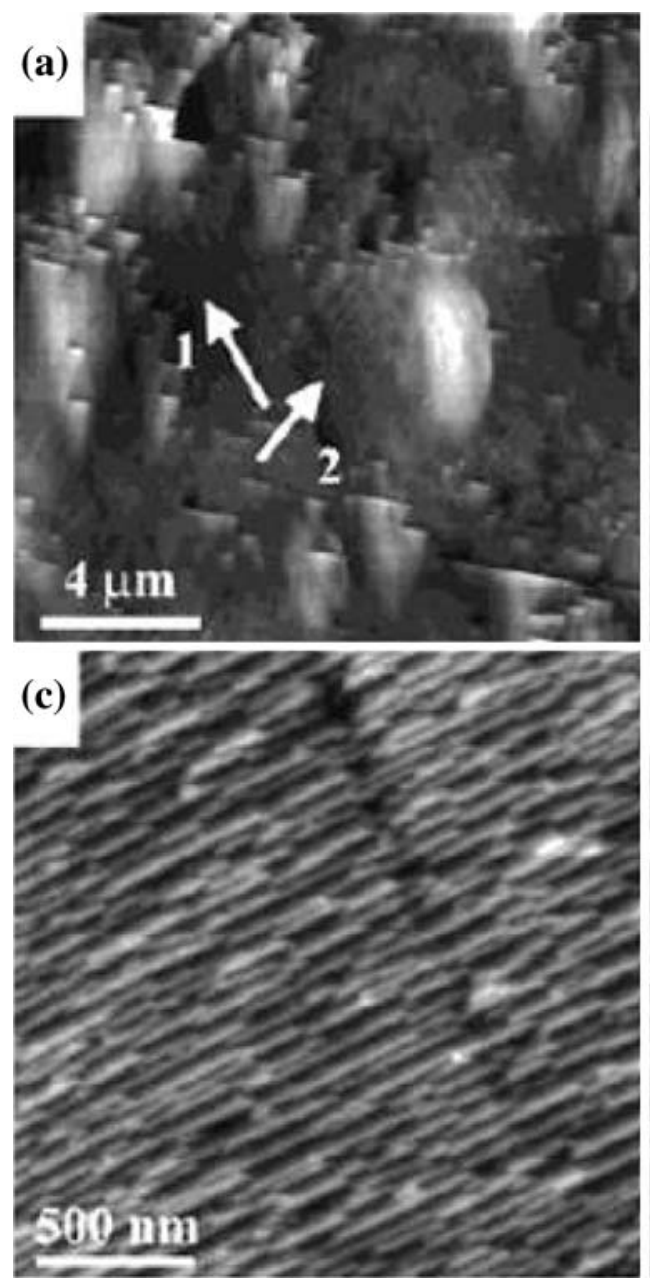
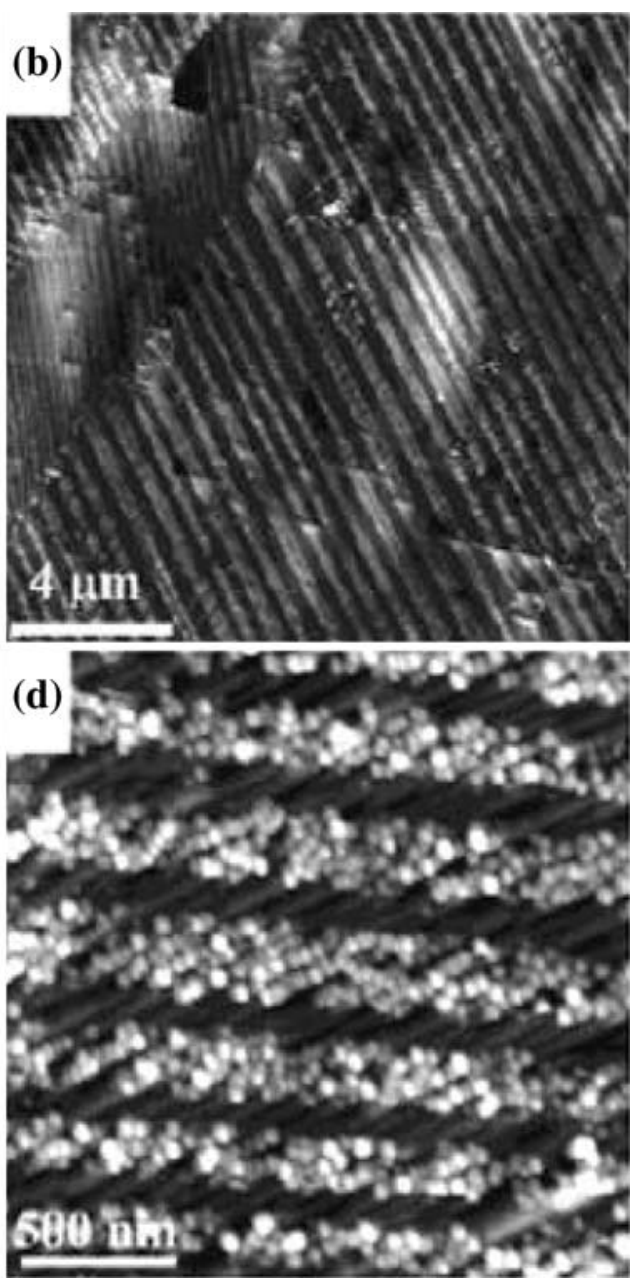

Fig. 3. Here the authors point out that there are several very interesting aspects to the growth of the silver on the positively poled domain.

The most important was that the $\mathrm{Ag}^{+}$ions should not be intimately attached to the $\mathrm{c}^{+}$surface and that in fact the negatively charged counter ions would be forming a Stern or double layer. Therefore, there has to be a mechanism that is allowing the movement of photoexcited carriers from the surface of the ferroelectric into the solution. This was thought to be tunnelling, that was allowing the electrons to pass through the bound ions and reduce the metal cations to metal. This hypothesis has recently been supported by some work by Jones and Dunn [34, 35] who have shown that there is an interaction between the Helmholtz plane and the species in solution available for reaction. Early work also showed that it was also possible to produce $\mathrm{PbO}$ by oxidation of lead salts on the negatively poled domains. The photoreduction and photo oxidation reactions on positive and negative domains are described by reactions 1 and 2, respectively.

$\mathrm{Ag}^{+}+\mathrm{e}^{-} \rightarrow \mathrm{Ag}^{0}$

$\mathrm{Pb}^{2+}+2 \mathrm{H}_{2} \mathrm{O}+2 \mathrm{~h}^{+} \rightarrow \mathrm{PbO}_{2}+4 \mathrm{H}^{+}$
There is a need for a 'salt-bridge' to be present to allow counter ions to migrate and retain charge neutrality during the electrochemical process-the full nature of the REDOX couple has yet to be verified and remains one of the outstanding questions in the field. However, recent detailed studies have shown that there is a complex relationship between position of the conduction and valence bands and the REDOX couple in solution that determines what chemistry occurs on the ferroelectric surface [36]. The intimate relationship between the incident flux of radiation means that the density and particle size of the deposited material can be controlled by varying the wavelength of irradiated ultraviolet light (as shown in Fig. 4a, b) [37]. The reasons for the variation in density and particle size with a variation in wavelength will be addressed in detail later article but, in summary here, stem from the interaction between the energy of the incident photon on the surface of the ferroelectric and the variation in the density of states that exist within the ferroelectric.

Using analogous tests it has been shown that the deposition of Rh, Pd, Au, Co, Ni, Fe can been achieved on PZT that has been grown via sol-gel. $\mathrm{BaTiO}_{3}$ was produced as a 

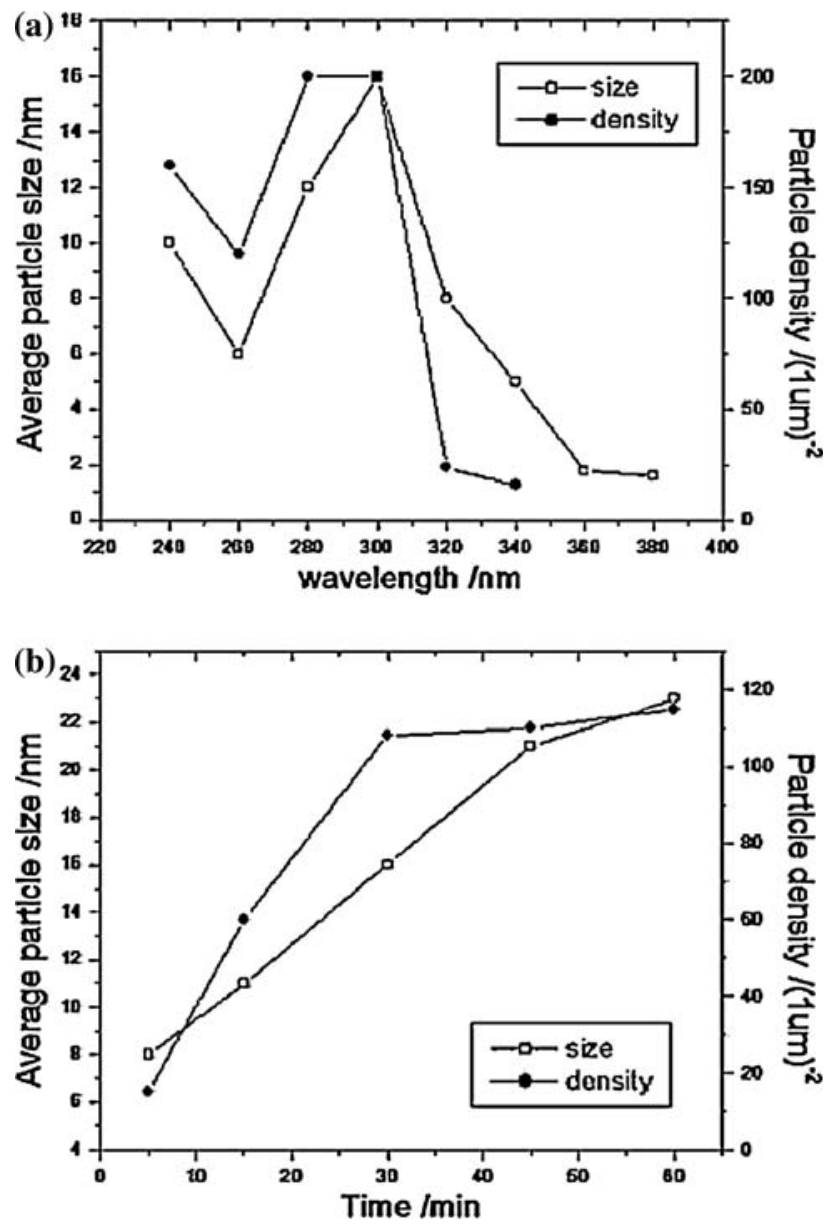

Fig. 4 a Wavelength dependence of particle size and density, $\mathbf{b}$ time dependence of particle size and density for Ag photoreduced from $10^{-4} \mathrm{M}$ (with permission from Ref. 37)

single crystal, thin film and polycrystals [32] and exhibited similar results. There is also now a growing body of literature showing that similar reactions can occur on $\mathrm{LiNbO}_{3}$, although there is not the same level of understanding for this material as the perovskite systems. In all these cases there has been a focus on the reduction of a metal salt to produce metallic products. If an examination of the band structure for PZT and $\mathrm{BaTiO}_{3}$ is performed then it is possible to see why reduction of certain metal salts is possible. The conduction band of the materials PZT and $\mathrm{BaTiO}_{3}$ sit between -0.5 and $-1 \mathrm{~V}$ when compared to the standard hydrogen electrode. The discrepancy of the exact location of the conduction band is due to variations in processing conditions, stoichiometry and experimental techniques. However, what has been determined is that salts with a reduction potential greater than the chemical potential of the conduction band will be reduced when the surface of the ferroelectric is under super band gap photoillumination. All of the metals that have been produced so far fit this criterion. The selective deposition process is attributed to the various phenomena taking place on the surface of ferroelectric thin films. In this review we will focus on the various properties and phenomena occurring on the surface of ferroelectric material.

\section{Ferroelectricity and piezoelectricity}

Ferroelectricity is a phenomenon in which a crystal exhibits two or more stable configurations in the absence of an electric field and can be shifted from one to another state by the application of electric field. The most studied and frequently applied ferroelectric materials are those with perovskite-type structures with general formula $\mathrm{ABO}_{3}$, which $\mathrm{A}$ and $\mathrm{B}$ are cations such as $\mathrm{Pb}^{2+} / \mathrm{Ba}^{2+} / \mathrm{Sr}^{2+}, \mathrm{Zr} / \mathrm{Ti}$ and $\mathrm{O}$ is oxygen anion $\mathrm{O}^{2-}$.

Piezoelectricity is a phenomenon in which a crystal can be polarised by the application of mechanical stress. Conversely when an electric field is applied across a piezoelectric crystal, it will change its dimensions to either expand or contract. Therefore, application of pressure to a piezoelectric crystal between two electrodes causes a charge to flow in one direction. The sign of piezoelectric charge or the direction of strain is the same as the direction of applied mechanical and electrical fields respectively.

\section{Domain and domain boundaries}

The regions of a ferroelectric crystal with uniformly oriented polarisation are called ferroelectric domains and the plane separating two domains is called a domain wall. Unpoled ferroelectric materials tend to form multiple domains because a single domain crystal would produce an electric depolarising field. This is very unfavourable from an energetic point of view and to produce uni-polar ferroelectric materials energy must be supplied in the form of the process of poling. Thus, during synthesis, a ferroelectric crystal forms into separate domains so that the equilibrium energy level between the domain walls and the electrostatic self-energy of the crystal is achieved [38]. It can be said that the general ferroelectric domain structures in equilibrium is a result of minimising the overall deformation and the electrostatic energy;

$W_{\text {tot }}=W_{\mathrm{M}}+W_{\mathrm{E}}+W_{\mathrm{DW}}+W_{\mathrm{S}}=\min$,

where $W_{\mathrm{M}}$ is the elastic energy, $W_{\mathrm{E}}$ is the electric energy, $W_{\mathrm{DW}}$ is the domain wall energy and $W_{\mathrm{S}}$ is the surface energy.

Those domains whose polarisation vector points in $+z$ direction are 'up' $\left(\mathrm{c}^{+}\right)$domains, domains whose polarisation vector points in $-z$ direction are 'down' $\left(\mathrm{c}^{-}\right)$domains 


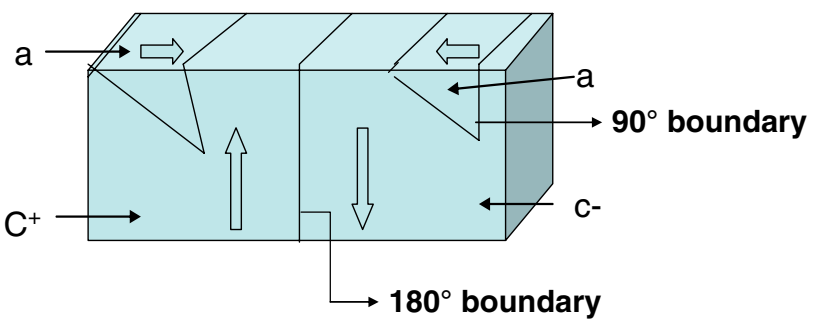

Fig. 5 Shows $\mathrm{c}^{+}, \mathrm{c}^{-}$and a domains in a ferroelectric crystal

and the domains in $\pm x$ or $\pm y$ direction, i.e. lies on the surface plane are lateral domains (a). The boundaries separating a-c domains are called $90^{\circ}$ boundaries, and those separating $\mathrm{c}^{+}-\mathrm{c}^{-}$domains are $180^{\circ}$ boundaries (the name implies the angle between the polarisation vectors in adjacent domains). Figure 5 depicts various domains and domain boundaries. In the tetragonal structure crystal, both 180 and $90^{\circ}$ domain walls can form in the crystal; however, the formation mechanism is slightly different. The formation of both 180 and $90^{\circ}$ domain walls can minimise the depolarisation field by compensating for the surface charge in an unpoled ferroelectric ceramics, but only the formation of the $90^{\circ}$ domain wall can release the elastic energy stored in the crystal. The motion and interaction of domain walls significantly affects the piezoelectric, mechanical and optical properties of the structure [39]. To be able to predict and control the domain wall kinetics, their physical and electrical properties need to be thoroughly understood and accurately determined.

In semiconducting ferroelectric thin films the coupling between polarisation and space charges leads to the formation of charge double layers at $90^{\circ}$ domain walls, which are decorated by defects such as dopants, oxygen vacancies [40]. There is no such charge layer formation in $180^{\circ}$ walls.

Over the years there have been extensive and exhaustive studies that have focused on the formation and static properties of domains in bulk crystalline ferroelectrics by polarising optical microscopy, etching and surface decoration [41, 42]. These methods have a disadvantage in that they have a low spatial resolution of the order of $1 \mu \mathrm{m}$. Under certain circumstances resolution below $10 \mathrm{~nm}$ can be achieved by SPM [27]. Domain related topographic features are characterised by contact and intermittent mode [43-48] and electrostatic properties above the surface by electrostatic force microscopy $[49,50]$.

\section{Ferroelectrics: insulators or semiconductors?}

Traditionally ferroelectrics have been regarded as insulators following the Devonshire-Ginsburg-Landau theory [51]. The band gap of ferroelectric materials is large, e.g. for PZT type materials it is around 3.2-3.7 eV [52-54], and for $\mathrm{BaTiO}_{3}$ it is around $3.2 \mathrm{eV}$. Variations in the band gap, especially for PZT and other complex perovskite materials, arise due to variations in processing conditions and other external factors and as such there is yet to be a definitive $E_{\mathrm{g}}$ that is widely accepter for PZT. Under equilibrium situations the materials can be treated as insulators, however, under the influence of sufficiently high energy radiation the non-equilibrium that develops follows basic semi-conductor theory. Furthermore the large concentration of structural defects acting as doping centres, the presence of Schottky barriers at contacts, and large leakage currents are characteristic properties of a semiconducting material. Therefore these materials can also be considered, and treated, as wide band gap semiconductors [55]. A nondoped PZT film, i.e. as close as possible to stoichiometric composition is a $p$-type semiconductor according to defect chemistry. Indeed the chemistry of the PZT produces some interesting effects; due to the oxygen vacancy accumulation an oxygen deficient layer, which is about $30 \mathrm{~nm}$ thick, $n$-type conductivity arises in the region close to the surface, whereas bulk remains $p$-type [56]. According to Mihara et al. [57] the $n$-type layer appears during the crystallisation annealing of films at high temperatures in the range 500 $700{ }^{\circ} \mathrm{C}$ because of the volatilisation of lead oxide from the film composition.

\section{Polarisation and screening}

In ferroelectric materials the displacement of a body centred cation, e.g. in a cubic perovskite $\mathrm{BaTiO}_{3}$ the displacement of $\mathrm{Ti}^{3+}$, gives rise to a dipole in the unit cell. The interactions between dipoles in unit cells cause polarisation alignment resulting in a polarisation bound charge at the surface of the block of ceramic. This polarisation charge affects surface topography, chemical reactivity, optical and electronic properties of ferroelectric surfaces and interfaces, and this is clearly demonstrated by phenomena such as ferroelectric electron emission [58, 59], polarisation dependent work function [60], metal photodeposition [32, 61].

On an oriented $\mathrm{BaTiO}_{3}$ crystal, the atomic polarisation may be directed either perpendicular or parallel to the plane of the surface whereas on an unoriented $\mathrm{BaTiO}_{3}$ crystal the atomic polarisation may be directed randomly. This atomic polarisation induces a surface charge $\sigma$ which is:

$\sigma=P \cdot n$,

where $\sigma$ is surface or polarisation charge, $P$ is polarisation vector, $n$ is unit vector normal to the surface. This induced surface charge may be compensated by free charge carriers and defects in the bulk (internal screening) and/or by the 


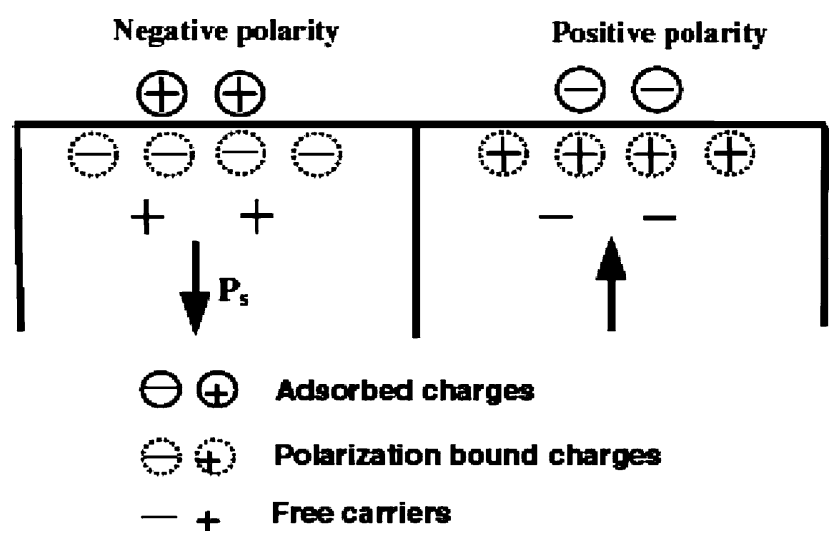

Fig. 6 A schematic diagram showing surface charges of a ferroelectric material. $P_{\mathrm{s}}$ is the spontaneous polarisation and the arrow represents the direction of spontaneous polarisation (with permission from Ref. 60)

surface adsorption of charged molecules from the environment (external screening) [16] as shown in Fig. 6.

\section{Surface layer}

The first suggestion about the existence of surface layer was given by Kanzig [62] on the basis of experiments performed on $\mathrm{BaTiO}_{3}$. It was observed that a strong electric field of $10^{4}-10^{6} \mathrm{~V} / \mathrm{cm}$ and a low dielectric constant could be observed in the volume of the surface layer. It was then shown that the surface layer is not a foreign film but is a surface region of the crystal in which dielectric saturation and piezoelectric compression occur because of strong electric field [63]. Through detailed investigation of properties and nature of the surface layer Treibwasser [64] modelled it as a Schottky barrier.

Further results have been completed to compare the electric potential, space charge density, defect density and polarisation in the surface layer to the bulk [40] of a ferroelectric material. It should be noted that there are expected significant differences in the behaviour of materials as they are reduced in thickness as the region of space layer approaches the thickness of the sample. In the case of PZT (30:70) samples approaching $50 \mathrm{~nm}$ in thickness will exhibit space charge characteristics almost throughout the sample thickness as the individual space charge layers at each interface are around $23 \mathrm{~nm}$ [65]. It is also interesting to note to that the stoichiometry of the sample can influence the space charge layer. However, this should not be counterintuitive as there is also a variation in the spontaneous polarisation as the stoichiometry changes. The measurements have been taken along the line $\mathrm{AA}^{\prime}$ in the $\mathrm{BaTiO}_{3}$ slab (as shown in Figs. 7 and 8). A depletion layer of approximately $100 \mathrm{~nm}$ thickness is observed near the $\mathrm{Pt}$ electrodes, accompanied by a voltage increase of $1.4 \mathrm{~V}$

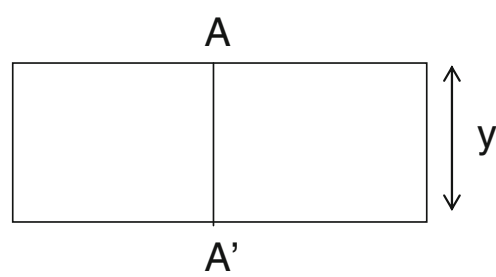

Fig. $7 \mathrm{~A} \mathrm{BaTiO}_{3}$ slab with $\mathrm{Pt}$ shorted electrodes on both faces
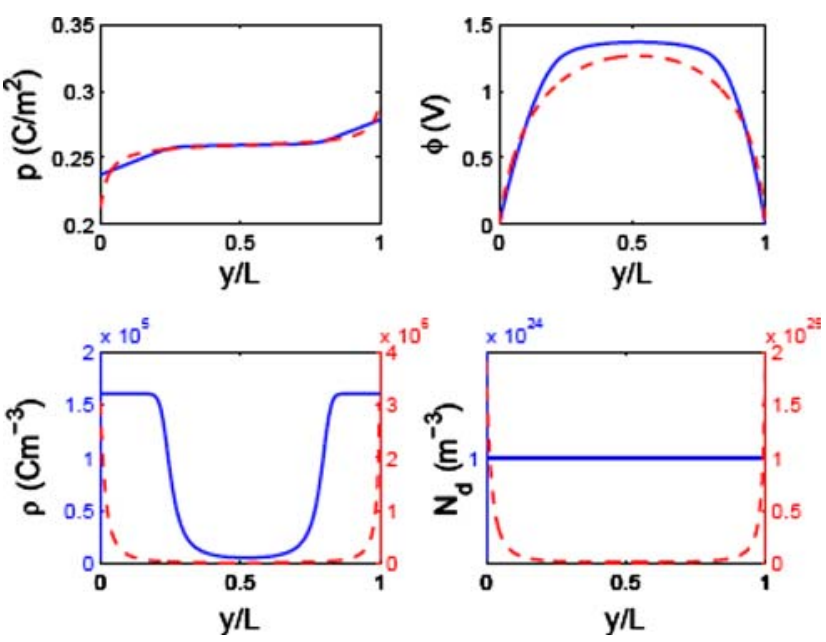

Fig. 8 The polarisation, electric potential, space charge density and defect density along the $\mathrm{A}-\mathrm{A}^{\prime}$ of the $\mathrm{BaTiO}_{3}$ slab in Fig. 6a. Solid lines without diffusion of defects; dashed lines with diffusion of defects (with permission from Ref. 40)

across the depletion layers [40]. The space charge density is at a maximum near the electrodes and is $\cong 0$ in the bulk. The large electric field in the depletion layer drives the diffusion of defects including oxygen vacancies, especially during annealing at high temperatures where the thermal energy enables the efficient movement of defects and ions into low energy positions within the crystal lattice.

In a ferroelectric material the width of the space charge layer is dependent on the composition of elements that constitute the material [65]. When investigating a set of metal- $\mathrm{Pb}\left(\mathrm{Zr}\right.$, Ti) $\mathrm{O}_{3}-$ metal samples with different $\mathrm{Zr} / \mathrm{Ti}$ ratios (from $92 / 8$ to 20/80) and variation in the width of surface layer $\delta$ along with other quantities like saturation polarisation $P_{\mathrm{s}}$, static dielectric constant $\varepsilon_{\mathrm{st}}$ hole concentration $p(T)$, apparent built-in potential $V_{\mathrm{bi}}{ }^{\prime}$, effective space charge density in the depleted layer $N_{\text {eff }}$, potential barrier $\phi_{\mathrm{B}}$ is observed. The evaluated values are shown in Table 1 .

The quality of the ferroelectric film is an important factor which contributes to the thickness of the surface layer. As defect density has an impact on the photochemical properties [66] of the ferroelectric material that has been processed in different ways could be expected to show some differing photochemical effects. When a sample has been made by processes such as sol-gel [67] then a very different defect density and structure through out the 
Table 1 The evaluated values for saturation polarisation $P_{\mathrm{s}}$, static dielectric constant $\varepsilon_{\mathrm{st}}$ hole concentration $p(T)$, apparent built-in potential $V_{\mathrm{bi}}{ }^{\prime}$, effective space charge density in the depleted layer $N_{\text {eff }}$, potential barrier $\phi_{\mathrm{B}}$ and width of the surface layer $\delta$ (with permission from Ref. 65)

\begin{tabular}{llllllll}
\hline PZT & $P_{\mathrm{S}}\left(\mu \mathrm{C} / \mathrm{cm}^{2}\right)$ & $\varepsilon_{\mathrm{st}}$ & $p(T)\left(10^{17} \mathrm{~cm}^{-3}\right)$ & $V_{\mathrm{bi}}{ }^{\prime}(\mathrm{V})$ & $N_{\text {eff }}($ average $)\left(10^{20} \mathrm{~cm}^{-3}\right)$ & $\phi_{\mathrm{B}}^{0}($ average $)(\mathrm{eV})$ & $\delta($ average $)(\mathrm{nm})$ \\
\hline $92 / 8$ & 6.1 & 247 & 3.8 & +0.10 & 0.42 & 1.21 & 1.21 \\
$65 / 35$ & 22.3 & 303 & 5.0 & +0.05 & 1.40 & 1.09 & 33.2 \\
$55 / 45$ & 31.1 & 438 & 2.2 & -0.10 & 0.34 & 1.37 & 13.3 \\
$30 / 70$ & 16.0 & 280 & 22 & -0.20 & 3.4 & 1.30 & 22.6 \\
$20 / 80$ & 40.5 & 180 & 50 & +0.40 & 17 & 3.0 \\
\hline
\end{tabular}

thickness of the thin film exists compared to MBE grown material. The interaction between the density and nature of defects, in the material and the internal depolarisation field leads to the development of the space charge layer. The depth to which the space charge layer penetrates is determined by how many mobile carriers are available to screen the depolarisation field. Therefore a material with a large spontaneous polarisation and few defects will generate a wide space charge layer, increasing the defect density, and with that the number of available carriers, reduces the width of the space charge layer [68]. If the ferroelectric is processed using low temperature sol-gel type mechanisms then there are different defect structures compared to material that has been processed at higher temperatures using standard ceramic processing techniques.

When dealing with materials at the nanoscale there is an interest in how the dimensions affect the fundamental properties of the material. This is also true for a ferroelectric and there has been some detailed work investigating the impact of cross sectional thickness on various parameters. Of particular interest here is the impact on the depolarisation of the ferroelectric and the influence that reducing the thickness of the thin film has. In a short review Scott [69] discusses finite size effects. It is well known that the spontaneous polarisation it at a maximum at the surface of the ferroelectric and decays to zero towards the centre. A key question is what is the dimension required for the spontaneous polarisation to decay and do nanostructured ferroelectrics allow this?

The influence of size on a ferroelectric when treated as a semi-conductor, can be seen in Table 1. The surface layer can vary from 33 to $3 \mathrm{~nm}$ depending on the composition (these values are for various PZT compositions). It is routine to make ferroelectric samples that are $10-100 \mathrm{~nm}$ in the $z$-direction. This would mean that for many ferroelectric compositions there would be an interaction between the SCL at both interfaces, as a result of the depolarisation field decaying across the thickness of the film. As a thin film ferroelectric would normally be grown on a conducting substrate there will be pinning of the Fermi levels of electrode and ferroelectric. In essence there is likely to be a strong influence of the depolarisation field on the surface photochemistry in thin film ferroelectric systems.

\section{Band bending}

The impact of an interface between a traditional semiconductor and solution or secondary phase has long since been established [70]. After the interface has come to equilibrium there has been a movement of free carriers that is the result of drift due to local concentration gradients. The band bending that is found in the semiconductor is a direct result of the drift of mobile carriers across the interface. There are, however, some fundamental differences when dealing with a material that has an internal field due to displacement of ions in the crystal lattice, in other words a ferroelectric material. These materials, while exhibiting $n$ or $p$-type behaviour do not generate bent bands in the same way as a traditional semiconductor-the need to screen the spontaneous depolarisation is too high and so the band bending is determined by this need.

The internal screening caused by free charge carriers or defects in the bulk causes 'band bending' at the surface of the ferroelectric material [71]. On a surface with positive polarity, the positive polarisation charge is screened by free electrons, negatively ionised acceptors or defects in the bulk and/or adsorption of negative ions from the air (as shown in Fig. 9). Similarly on the surface with negative polarity the negative polarisation charge is screened by free holes, positively ionised donors, defects and/or adsorption
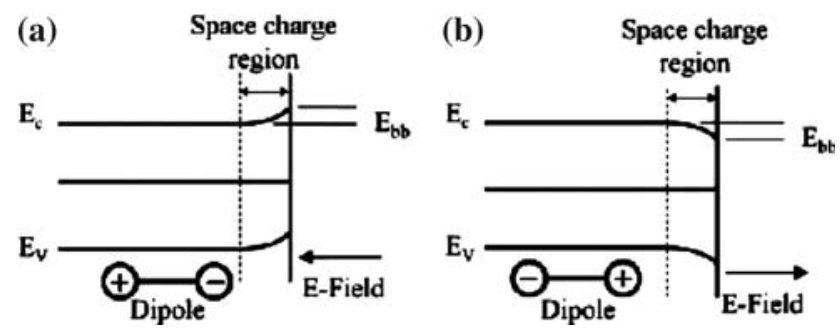

Fig. 9 Schematic diagram of band bending in the ferroelectric perovskite $\mathbf{a}$ a surface with negative polarity ' $\mathrm{c}^{-}$, and $\mathbf{b}$ a surface with positive polarity ' $\mathrm{c}^{+}$' (with permission from Ref. 85) 
of positive ions from the atmosphere. The intrinsic screening gives rise to an internal electric field which causes band bending in the near surface region, resulting in the formation of depletion and accumulation layers, as shown in Fig. 9.

It is generally true that for perovskites such as $\mathrm{BaTiO}_{3}$ and $\mathrm{PbTiO}_{3}$ as well as the more complex systems such as $\mathrm{PbZr}_{x} \mathrm{Ti}_{(1-x)} \mathrm{O}_{3}$ (PZT) that $E_{\mathrm{v}}$ is the top of the valence band and is associated with oxygen 2 p orbitals and $E_{\mathrm{c}}$ is bottom of conduction band associated with titanium, or A/B $3 \mathrm{~d}$ orbitals. $E_{\mathrm{f}}$ is the Fermi level and $E^{\mathrm{bb}}$ is the energy associated with band bending that is introduced either due to the field developed due to crystal distortion associated with ferroelectric behaviour or contact with. The density of states for the ferroelectric materials varies with the composition and also the processing parameters of the materials.

\section{Electrostatic properties of the ferroelectric surface}

The electrostatic properties of the ferroelectric surface play a significant role in its behaviour under various conditions. As the polarisation charge is always screened when at equilibrium, and in any environment that is not high vacuum, through a variety of mechanisms a screening charge equivalent to surface charge density is present. This screening charge is of opposite polarity to the polarisation charge. In this section we will discuss the following cases that have been previously highlighted as applicable to the surface of a ferroelectric material [72];

1. Completely unscreened surface $\sigma_{\mathrm{s}}=0$.

2. Partially screened surface $\sigma_{\text {pol }} \geq \sigma_{\mathrm{s}}$.

3. Completely screened surface $\sigma_{\text {pol }}=-\sigma_{\mathrm{s}}$.

4. Over-screened surface $\sigma_{\text {pol }} \prec-\sigma_{\text {s }}$.

A completely unscreened surface is a very unfavourable energy state. This state is unlikely to occur under ambient conditions and can be observed only in vacuum. This is due to the presence of water in all normal circumstances and the associated screening through interactions with the dipoles in the water and development of stable interface layers. There will also be a number of ionic constituents in water on the ferroelectric surface that are a result of partial dissolution of the ferroelectric or in the water itself. These components will interact with the surface of the ferroelectric to form a Helmholz layer to further screen the surface charge. Over-screened surfaces may occur and have been observed during bias induced domain switching [73] but are not likely in ambient conditions at equilibrium. The most likely state of ferroelectric surfaces, when in ambient environments such as in air and at equilibrium, is the partially or completely screened surface. This is due to the adsorption of water molecules and salts on them as described to refute the possibility of an unscreened surface. The charge distribution on this type of surface can be given in terms of the uncompensated charge component: $\Delta \sigma=\sigma_{\mathrm{pol}}-\sigma_{\mathrm{s}}[$ [72]. The results that have been obtained for potential and electric field measurements on unscreened and completely screened surfaces are shown in Fig. 10. The non-contact measurements have been performed with the tip surface separation set to between 10 and $100 \mathrm{~nm}$. At this distance from the surface the electrostatic interaction of the material being investigated can be accurately measured and recorded. Therefore the impact of screening on the ferroelectric surface can be determined.

The sign and magnitude of surface potential on a ferroelectric surface are not only dependent on polarisation charges, but they depend on the intricate balance between the polarisation and screening charges. An extreme case could be surface polarisation reversal due to the sorption of highly polarised long chain hydrocarbons on the surface of a ferroelectric akin to the behaviour of detergent molecules in an oil and water mix. In the case of the ferroelectric the balance is due to the availability of carriers (from within the sample) and the variation in the space charge layer that forms. The exact shape and dimensions of the space charge layer depend on a number of factors that are material and in some cases sample specific. It has been shown that photo deposition of silver can occur on both positive and negative domains on PZT when it is [100] orientated, but that silver only deposits on PZT [111] when it is positively charged [74]. This is due to variations in the density of states and also in the variation in the remnant polarisation of the sample, which impacts the shape of the space charge layer and screening processes. It is clear from Fig. 10 that above the unscreened surface the surface potential varies linearly and reciprocally with domain size whereas the electric field is domain size independent. Above the completely screened surface the electric field varies linearly and reciprocally with domain size and the surface potential is domain size independent.

The surface potential measured by scanning surface potential microscopy [75] on a ferroelectric surface is smaller and of opposite sign than the polarisation charge [76].

\section{Electronically active grain boundaries}

The grain boundary (GB) is a region which separates crystallographically coherent areas in crystalline solids. GBs act as barriers for the charge transport of carriers in materials showing ionic/electronic conduction. The GB effect has been studied for a variety of $n$-type semiconducting ceramics [77, 78]. For $n$-type semiconducting ceramics negatively charged GB states are compensated by 
Fig. 10 a An unscreened surface, b a completely screened surface, $\mathbf{c}$ and e potential and electric field in the vicinity of unscreened surface, $\mathbf{d}$ and $\mathbf{f}$ potential and electric field in the vicinity of completely screened surfaces (with permission from Ref. 72) (a)

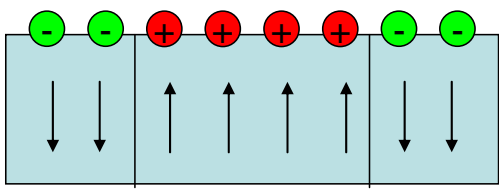

(c)

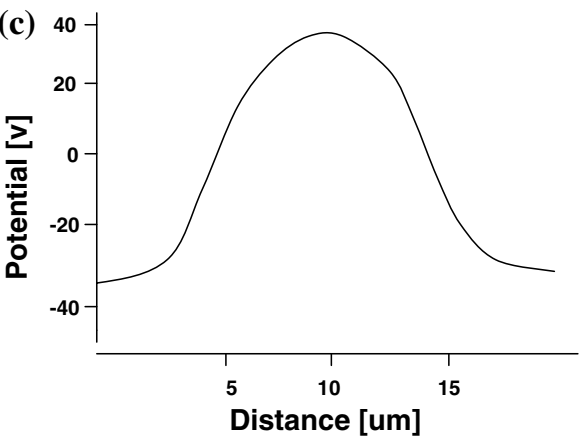

(e)

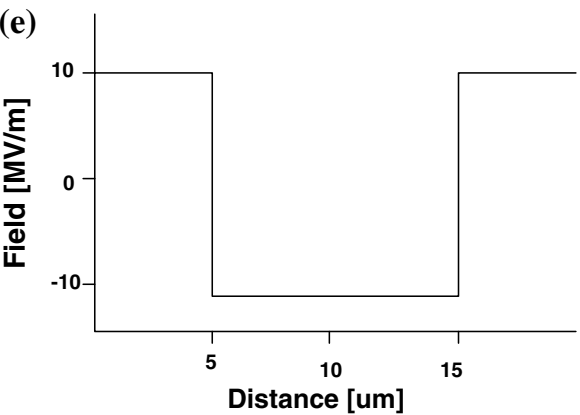

(b)

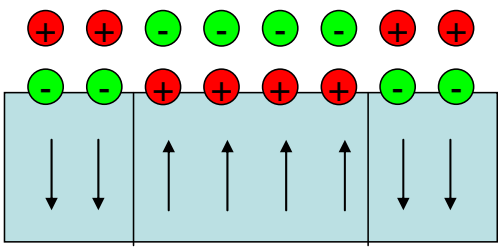

(d)
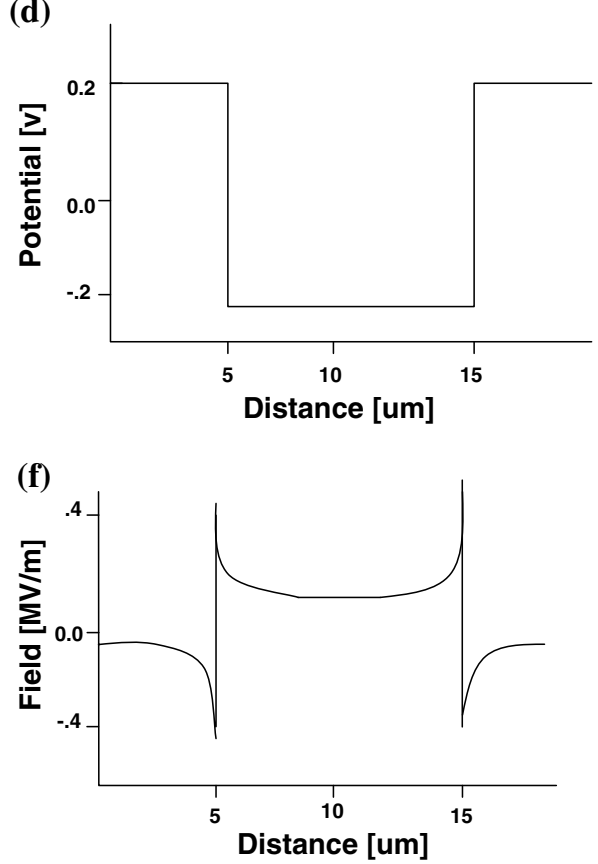

positively charged donor centres in the bulk close to the GB. This creates a depletion of the negatively charged mobile carriers in a layer of the width $d_{\mathrm{GB}}$ symmetrically surrounding all grain boundaries, and leads the formation of depletion layers. From the electronic point of view these depletion layers can be described as two Schottky diodes back to back. In perovskite type alkaline earth titanates a highly resistive GB layer has been observed [79-81]. In acceptor, $p$-type, doped alkaline earth titanates the GB states are electrically active and are positively charged due to donor centres. These are compensated by negative space charged acceptor dopants from the bulk close to the GB. The positive charge in the GB creates a depletion region of mobile positively charged carriers, holes and oxygen vacancies. This depletion space charge layer can be considered as two back to back Schottky barriers. This model was introduced by Chiang and Tagaki [82].

With the core of the GB heavily positively charged, the outer region becomes depleted of electrons. This gives rise to a depletion region around the GB core and causes band bending at the GB as shown in Fig. 12. The depletion region has a space charge which is greatest at the core of the GB and reduces as it moves into the grains [83, 84]. The impact of grain boundaries on the semi-conducting nature of a ferroelectric have recently been studied by
Jones and Dunn [85] using UV irradiation of PZT surfaces and the growth of Ag clusters through reduction of silver cations. The grain structure of highly heterogeneous PZT(30/70) was found to have a significant impact on the rate of growth of sliver clusters, see Fig. 11. During UV irradiation electron-hole pairs are generated in the film due to interactions with incoming photons. The combination of band bending and positive electric field present at the GB causes the electrons to migrate towards it as shown in Fig. 12. Due to this migration there is an increased availability of electrons for metal reduction at the grain boundaries than at the surface of the grain away from the boundaries. A table of the relative rates of deposition can be seen in Table 2 .

\section{Ferroelectric photocurrents and surface photochemistry}

Conduction in solids is dependent upon the mobilities and concentrations of both ionic defects (interstitial ions and vacancies) and electronic charge carriers (electrons and holes) through the bulk and along and across interfaces such as grain boundaries, electrode contacts and surfaces [86]. It has been shown by Maier [86] in 1993, that both 
Fig. 11 AFM image (left) of rosette structure of PZT grown on indium tin oxide and corresponding PFM image (right). There are an anomalous number of silver nanoparticles at the interface between the grains that have grown independent of the local domain structure of the PZT (with permission from Ref. 85)
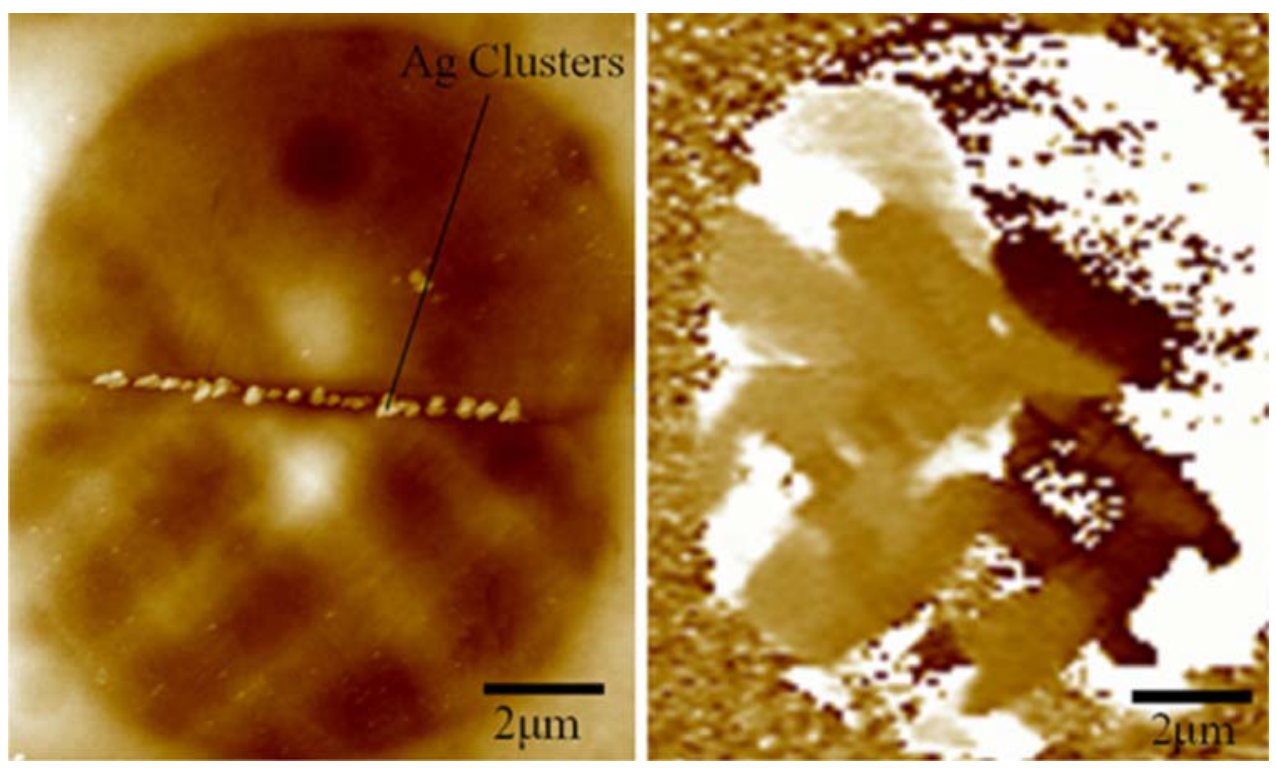

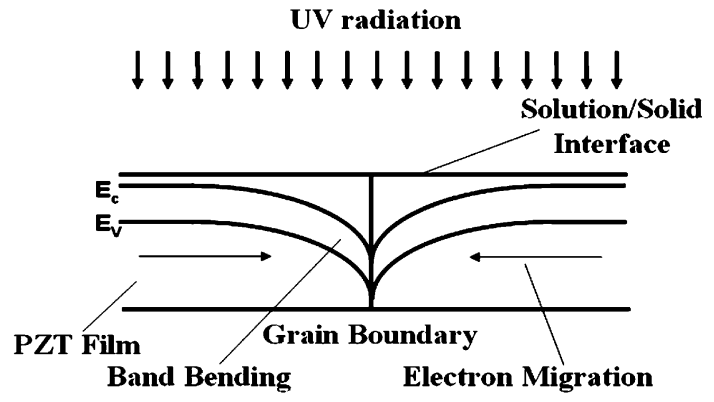

Fig. 12 Flow of electrons in the PZT at grain boundaries during UV irradiation (with permission from Ref. 85)

Table 2 Relative rates of deposition of Ag clusters on a highly heterogeneous PZT surface (with permission from Ref. 85)

\begin{tabular}{ll}
\hline Rank & Boundary type \\
\hline 1 & Grain boundaries \\
2 & Positive domains \\
3 & Interphase boundaries \\
3 & Domain boundaries \\
\hline
\end{tabular}

conductive mechanisms: ionic and electronic, may be modelled using analogous methods and that both mechanisms, and their dependence on environmental parameters such as temperature and partial pressure, participate in the overall reactivity of many materials, including ferroelectrics.

Electronic conduction can be activated through the optical transition of electrons from valence to conduction band but, as quantised light particles. Incident photons must have energies exceeding that of the material's band gap. This limiting factor is referred to as the fundamental absorption and in $\mathrm{BaTiO}_{3}$ for example, only energies greater than the band gap $\left(E_{\mathrm{g}}\right)$ value of $\sim 3.15 \mathrm{eV}$ at room temperature [87] are productive, with each photon producing one electronic charge $(q)$ at a voltage of:

$V_{\mathrm{g}}=E_{\mathrm{g}} / q=h v_{\mathrm{g}}$

where $h$ is Planck's constant and $v_{\mathrm{g}}$ the band gap frequency. Therefore, the beneficial range of incident spectral wavelengths is limited by the value of the band gap.

Ionic conduction mechanisms rely on the transport of interstitial ions and vacancies which are also subject to a minimum energy requirement that is referred to as free activation energy. It has been shown by Franklin [88] that the enthalpies of vacancy formation in elemental solids are approximately proportional to their absolute melting points with results ranging from a fraction of one $\mathrm{eV}$ (e.g. Ar, Xe) to over $2 \mathrm{eV}$ (Mo), whilst the free enthalpy change caused by the introduction of an oxygen defect is in the order of $1 \mathrm{eV}$, or $100 \mathrm{~kJ} \mathrm{~mol}^{-1}$ [89].

Defect concentrations can be manipulated via thermal excitation or doping and the introduction of intermediate donor or acceptor impurity levels within the band gap can influence the level of energy required for electron and hole transitions across the gap [90]. These intermediate states can behave as non-radiative Schockley Hall Read (SHR) generation-recombination centres or Auger type traps.

While SHR generation-recombination enables electrons and holes to recombine and thus navigate the potential barrier to produce an additional current flow mechanism via thermal exchange, recombination at deep Auger trap sites is non-radiative and reduces carrier densities whilst trapping the energy within deep lying energy bands. However, Auger rates decrease exponentially with increasing bandgap values and become more or less inconsequential for bandgaps above $\sim 1.5 \mathrm{eV}$ [91]. It 
should also be noted however that heavy doping results in a narrowing of the bandgap.

\section{Kinetics}

In all photochemical reactions the kinetics of the process plays an important role. While the thermodynamics of the system may be favourable the rate at which the products are formed is determined by the complex interaction of the surface and near boundary layers. As has been discussed earlier this is very complex for a ferroelectric surface that is screened by counter ions as the inherent REDOX couple (e.g. reduction on a $\mathrm{c}^{+}$ surface) requires the participation of $\mathrm{M}^{+}$species in solution. The development of a screening layer that is composed of species that are effectively of an opposite charge to those required for the reaction means that any REDOX chemistry must occur through either local tunnelling or a disruption of the local double layer interface [92]. What is interesting is that although there are a number of inhibiting features to the REDOX chemistry at the surface of a ferroelectric there is also a strong driving force - the internal dipole - that enables the reactions to proceed. It has been shown that reduction of highly charged cations with large negative reduction potentials can be achieved on ferroelectric surfaces, an example of which is the reduction of $\mathrm{Al}^{3+}$ to $\mathrm{Al}^{0}$ on $\mathrm{LiNbO}_{3}$.

It is possible to describe the reactions occurring on the surface of the ferroelectric using classical interpretations of the kinetics at an interface. As demonstrated by Bard and Faulkner [93], for a classic metal electrode reaction:

$\mathrm{O}+n e \underset{K_{\mathrm{b}}}{\stackrel{K_{\mathrm{f}}}{\longrightarrow}} \mathrm{R}$

where $k_{\mathrm{f}}$ and $k_{\mathrm{b}}$ are the rate constants of the forward and backward reactions, respectively, $n$ is the electron concentration and $e$ the electron charge. At equilibrium, this system is characterised by the Nernst equation:

$E=E^{0^{\prime}}+(R T / n F) \ln \left(C_{\mathrm{O}}^{*} / C_{\mathrm{R}}^{*}\right)$

where $E^{0 \prime}$ is the formal potential (a calculated value for each half cell measured across the complete cell), $F$ is Faraday's charge on one mole of electrons, and $C_{\mathrm{O}}^{*}$ and $C_{\mathrm{R}}^{*}$ the bulk concentrations of oxidation and reduction species.

The overall current across a cell $(i)$ under external bias is the sum of both forward cathodic $\left(i_{\mathrm{c}}\right)$ and backward anodic $\left(i_{\mathrm{a}}\right)$ currents and is dependant upon the surface area $(A)$ and surface concentrations at distance from the surface 0 and time, $t$, i.e. $C(0, t)$

$i=i_{\mathrm{c}}-i_{\mathrm{a}}=n F A\left[k_{\mathrm{f}} C_{\mathrm{O}}(0, t)-k_{\mathrm{b}} C_{\mathrm{R}}(0, t)\right]$

In heterogeneous materials, such as metal-semiconductor junctions, this kinetic behaviour is influenced by interfacial potential differences as previously discussed, and influenced by the development of local double layer interactions. The dependence of the rate constants $k_{\mathrm{f}}$ and $k_{\mathrm{b}}$ on these influences can be expressed as:

$k_{\mathrm{f}}=k^{0} e^{-\alpha n f\left(E-E^{0^{\prime}}\right)}$

$k_{\mathrm{b}}=k^{0} e^{(1-\alpha) n f\left(E-E^{0^{\prime}}\right)}$

where $f=F / R T$, and $k^{0}$ and $\alpha$ are adjustable, phenomenological parameters evaluated by experiment called the intrinsic or standard rate constant, which is the value of $k_{\mathrm{f}}$ and $k_{\mathrm{b}}$ at $E^{0^{\prime}}$, and the transfer coefficient which is a measure of symmetry of the energy barrier:

$\alpha=\tan \theta /(\tan \Phi+\tan \theta)$

thus, for symmetrical intersections $\Phi=\theta$, and $\alpha=1 / 2$.

As previously mentioned however, most chemical processes occur over multiple steps. In cases where one of these steps is slower than the others, this step controls the overall process rate and as such is the rate-determining step, for example in perovskites, such as $\mathrm{BaTiO}_{3}$, holes have particularly slow mobilities [94] and are less likely to tunnel through any barrier such as that presented by a double layer. In these situations the number of electrons effected by the rate-determining step $\left(n_{\mathrm{a}}\right)$ must be distinguishable from the overall number of electrons involved in the overall process of converting one mole of reactant $(n)$, where $n_{\mathrm{a}} \leq n$ :

$i=n F A k^{0}\left[C_{\mathrm{O}}(0, t) e^{-\alpha n a f\left(E-E^{0^{\prime}}\right)}-C_{\mathrm{R}}(0, t) e^{(1-\alpha) n a f\left(E-E^{0^{\prime}}\right)}\right]$

For non-equilibrium potentials, boundary resistance or overpotential $(\eta)$ is produced by polarisation (nonspontaneous) and mass transfer pseudoresistances:

$\eta=-i\left(R_{\mathrm{ct}}+R_{\mathrm{mt}, \mathrm{c}}+R_{\mathrm{mt}, \mathrm{a}}\right)$

where $R_{\mathrm{ct}}$ is the charge transfer resistance and $R_{\mathrm{mt}, \mathrm{c}}$ and $R_{\mathrm{mt}, \mathrm{a}}$ the mass transfer resistance for anodic and cathodic ions, respectively.

The reaction rate $(v)$ at an electrode surface is proportional to the faradaic current:

$v\left(\mathrm{~mol} \mathrm{~s}^{-1} \mathrm{~cm}^{-2}\right)=i / n F A=j / n F$

Thus, surface reactivity tends to be determined via currentvoltage $(I-V)$, capacitance-voltage $(C-V)$, or photoresponse-wavelength $(I-\lambda)$ characteristics. This result shows why in the case of a poled ferroelectric material it is possible for the ferroelectric dipole to influence the surface photochemistry. The local field in the ferroelectric can influence (either beneficially or adversely depending on the dipole) the photocurrent that is generated. 


\section{Surface photochemistry}

For surface electrochemistry to take place the standard reaction potential $\left(U^{0}\right)$, corresponding to the sum of the energy requirements for the transfer of the participating defect species, must be exceeded to accommodate the fugacities of the products and the overvoltages of the electrodes. For semiconducting electrodes, the Fermi level of the electrode corresponds to its surface potential. Thus, the flat-band potential $\left(U_{\mathrm{fb}}\right)$ of the electrode, which is itself sensitive to the $\mathrm{pH}$ of the electrolyte at an approximate rate of $0.059 \mathrm{~V}$ per $\mathrm{pH}$ unit [95], and to absorption of certain solutes $[96,97]$ must exceed the required reaction potential and will define the 'activation' or 'switch on' potential of the reaction.

For example, as Fushima and Honda [13] in 1972 first demonstrated, the decomposition of water requires a potential increase of $1.23 \mathrm{~V}$ at the anodic surface to manifest oxidation $(\mathrm{O})$, corresponding to the transfer of two electrons from oxygen to hydrogen. If the reaction were carried out in a single absorption step, for a typical metalsemiconductor electrode this reaction can be calculated to require photons with energies exceeding $\sim 1.48 \mathrm{eV}$. However, as Tomkiewicz and Fay [98], in 1979 note, although $60 \%$ of solar radiation meets this criterion, water is not transparent in the extreme spectral regions and so an additional agent, or sensitiser, is used as a reaction intermediary, absorbing solar energy and delivering it to the water creating a stepped reaction, see Fig. 13.

In general therefore, photolysis may be optimised via manipulation of both the chemical and electrical electrode surface potentials.

In a typical cell configuration, using an $n$-type photoanode and a $p$-type photocathode, overall cell efficiency is limited by the least efficient electrode. Under short circuit operation, such as would be the case in a 'wireless' cell, the overall energy requirements may be summarised:

$$
\begin{aligned}
& \left(U_{\mathrm{fb}}\right)_{p}>\left(U_{\mathrm{fb}}\right)_{n} \\
& \left(E_{\mathrm{g}}-E_{\mathrm{FV}}\right)_{p}-\left(E_{\mathrm{g}}-E_{\mathrm{FC}}\right)_{n}>e\left(x+V_{\mathrm{B} p}+V_{\mathrm{B} n}\right)
\end{aligned}
$$

where $V_{\mathrm{B} p}$ and $V_{\mathrm{B} n}$ are the band bending voltages at the $p$ and $n$ semiconductors, respectively, $x$ is the required reaction potential and the inequality expressed in Eq. 16 equals the sum of the overvoltages at the two interfaces.

\section{Ferroelectric photocurrents and the anomalously high photovoltage}

It is well know that for a standard semiconductor the photovoltage cannot exceed the band gap of the semiconductor. In practice several features combine to mean that the photovoltage is lower than the band gap of the semiconductor, these include parasitic losses, and losses associated with local defects in the semi-conductor. The only exception has been semiconductor heterostructures that have been textured to give a series of $p-n$ junctions which break this fundamental rule. One group of materials that break this rule when treated homogeneously are ferroelectric materials. Anomalously high photovoltages (APV) have been reported from ferroelectric Rochelle salt crystal as early as 1939 by Brady and Moore [99]. It has been shown that APV's in the order of $40 \mathrm{~V}$ can be obtained from $\mathrm{BaTiO}_{3}$ [100] under ideal circumstances which far exceed the band gap of $3.2 \mathrm{eV}$. The source of the APV comes from an interaction of phase transition, internal dipole and interaction with the incoming radiation.
Fig. 13 Conceptual diagram for a water splitting system, showing energy transfer requirements of the participating species, using a two steps to include intermediary donor/acceptor redox shuttle and $\mathbf{b}$ one step (with permission from Ref. 124)

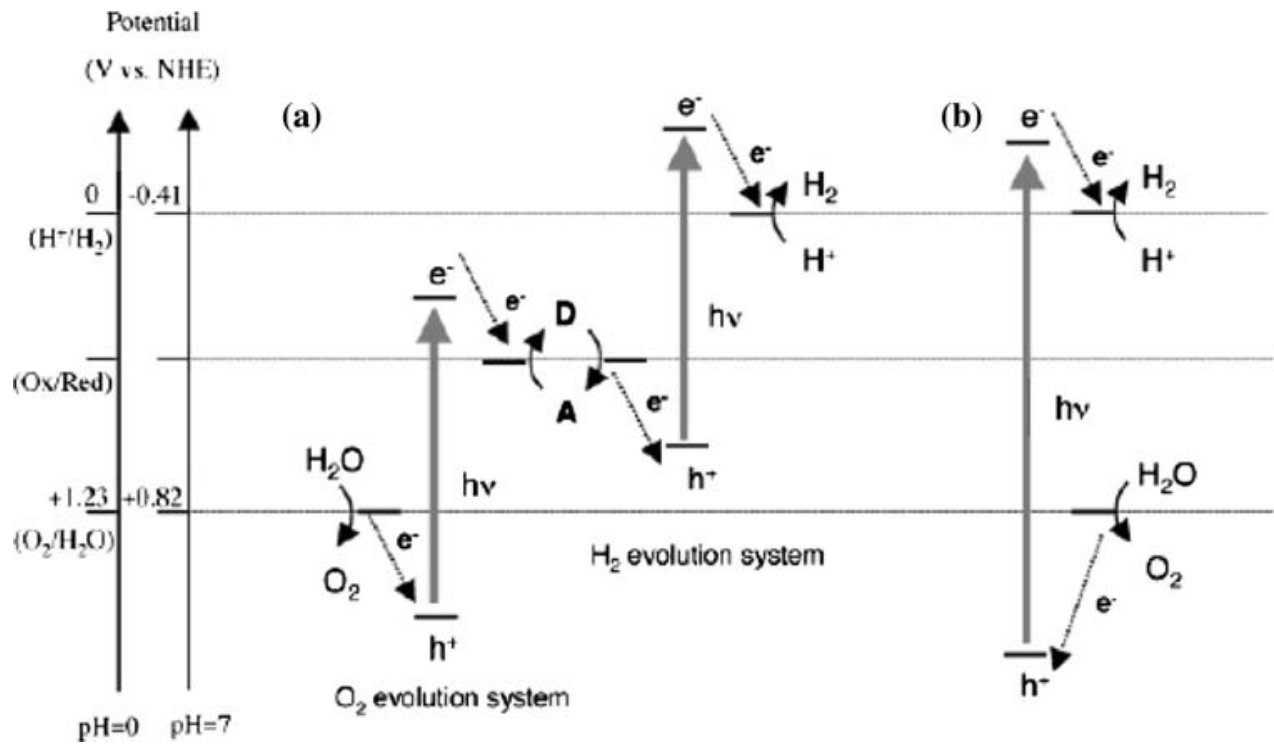


Later, whilst researching barium titanate and lithium niobate, respectively, both Chynoweth [101] in 1955 and Chen [102] in 1967 recorded weak but steady, underlying photocurrents in each ferroelectric crystal when under illumination with no external bias and heated through temperatures rising from below, to well above, their individual Curie temperatures. Open circuit voltages were eventually measured in single domain barium titanate crystal [103] and found to be fractional relative to the $\sim 3.15 \mathrm{eV}$ band gap [104]. Similarly less than band gap photovoltages were also observed in unpoled ceramic barium titanate [105]. These photovoltages were linked to the presence of potential variations within the crystal, supporting Kanzig's [62] in 1955 proposal that there exists temperature independent, space charge or barrier layers at crystal surfaces induced by extrinsic ionic conduction at impurity concentrations nearing $10^{18} \mathrm{~cm}^{-3}$. According to Kanzig, in ferroelectric crystals fields of $10^{5}$ or $10^{6} \mathrm{~V} \mathrm{~cm}^{-3}$ are enough to generate saturation polarisation within this surface layer, creating a potential difference of approximately $1 \mathrm{~V}$ between surface and bulk, and the resulting piezoelectric strain is structurally inelastic, prevailing despite bulk phase changes resulting from above Curie temperatures.

In contrast, Glass et al. [106] reported high photovoltages in doped single crystal $\mathrm{LiNbO}_{3}$ and Brody later reported greater than band gap photovoltages of several hundred volts per centimetre in poled ceramic $\mathrm{BaTiO}_{3}+5 \mathrm{wt} \% \mathrm{CaTiO}_{3}, \mathrm{~Pb}\left(\mathrm{Zr}_{0.65}, \mathrm{Ti}_{0.35}\right) \mathrm{O}_{3}$ and other ferroelectric ceramics [107-109] which were shown to be linearly proportional to both the sample length and the remnant polarisation and were observed only below the Curie temperature. Ionue et al. [110] recorded photovoltages three times larger than the bandgap for Lead Strontium Zirconate Titanate with directed polarisation fields in opposite directions to the photocurrent.

A mechanism, based on similar behaviour observed in semiconductor materials such as thin polycrystalline semiconductor films of Cadmium telluride [111] and striated zinc sulphide crystals [112], where the potential differences across individual crystallites, having the same origins as single crystal surface barrier layers, create multiple smaller than band gap voltages that add up in series across the whole was proposed, in 1975, by Brody and Crowne [89] with the total voltage written as:

$V=\left(P_{\mathrm{s}} S N_{0}\right) / \varepsilon_{\mathrm{s}}\left[1+\varepsilon_{\mathrm{b}} S / \varepsilon_{\mathrm{s}}(L-2 S)\right]$

where $P_{\mathrm{S}}$ is the spontaneous polarisation, $S$ is the length of the space charge region, $N_{0}$ is the number of crystal grains, $\varepsilon_{\mathrm{S}}$ is the dielectric constant of the space charge region, $\varepsilon_{\mathrm{b}}$ is the dielectric constant of the bulk and $L$ is the particle size.

\section{Ferroelectric photochemistry}

Following Fujishima and Honda's demonstration that $\mathrm{TiO}_{2}$ could be used as a photocatalyst without itself decomposing [13], others went on to demonstrate similar reactive stability in further metal oxide electrodes such as $\mathrm{SnO}_{2}$ [113] and $\mathrm{WO}_{3}$ [114], until in 1975 Mavroides et al. [115], closely followed in 1976 by Wrighton et al. [116] reported small currents resulting in $\mathrm{H}_{2}$ evolution at $\mathrm{SrTiO}_{3}$ electrodes under zero applied voltage in basic electrolytes. In the same year Nasby and Quinn [14] and a Japanese team, Okuda et al. [117] went on to compare the behaviour of single crystal and ceramic $\mathrm{BaTiO}_{3}$ and $\mathrm{SrTiO}_{3}$ electrodes, respectively, reporting similarly repeatable and stable current-voltage characteristics with both single crystal and ceramic electrodes producing small photoinduced currents under short circuit conditions. Both teams reported that $\mathrm{SrTiO}_{3}$ was more photosensitive than $\mathrm{BaTiO}_{3}$. Nasby and Quinn [14] also noted that their results demonstrated "the blocking character of depletion layer formation indicated by negligible dark current with anodic polarisation", and that increasing the $\mathrm{pH}$ of the electrolyte increased the turnon voltage under illumination, dopant concentrations and flat-band potential indicating the applicability of the MottSchottky relation:

$E=E^{0^{\prime}}+(R T / n F) \ln \left(C_{\mathrm{O}}^{*} / C_{\mathrm{R}}^{*}\right)$

By 1985 Inoue et al. were experimenting with ferroelectric substrates such as $\mathrm{LiNbO}_{3}$ [118], PZT and $\mathrm{BaTiO}_{3}$ [119] beneath $\mathrm{Pt} / \mathrm{TiO}_{2}$ thin films producing semiconductormetal-ferroelectric junctions attempting to combine the reactivity of $\mathrm{TiO}_{2}$ with the spatial separation of charge carriers provided by the ferroelectric substrate. They showed that film conductivity increased to a maximum at thicknesses of 50-100 nm while any influence of the underlying perpendicularly poled ferroelectric substrate vanished for film thicknesses above $200 \mathrm{~nm}$. For film thicknesses of $<50 \mathrm{~nm}$ the semiconductor carrier densities are too low to be affected by the polarisation field. Polarisation-direction dependence, with positively polarised surfaces facilitating photoexcited semiconductor electron transport towards chemically active sites on the Pt metal and negatively polarised substrates opposing this outcome and therefore producing negligible photoconductivity, was directly attributed to the presence of anomalous photovoltaic effects. The influence of poled ferroelectric substrates was shown to disappear as a result of phase transition above their Curie temperatures [35]. In 2002 Kalinin et al. [1] carried out research that supported the significance of semiconductor properties in the ferroelectric $\mathrm{BaTiO}_{3}$ during the photodeposition of metals. 
Hanson et al. [66] published work showing that the local reactivity on ferroelectric surfaces depends on relative densities of polarisation charges, adsorbed charges and surface states as well as the polarisation itself. Photochemical reduction of silver ions on the surface of a domain-patterned $\mathrm{LiNbO}_{3}$ template resulted in silver decoration of $180^{\circ}$ domain walls rather than of positive domains as would be expected. This effect was explained by Hanson et al. as a combination of inhomogeneous distribution of the electric field in the vicinity of the domain wall and low conductivity of the template material. Dunn et al. [35] have proposed that extreme band bending due to a build up of charge at the surface, can bring the flat-band region close enough to the surface to allow carriers to tunnel through the space charge region and thus reach active reaction sites.

Indeed the interest in ferroelectric photochemistry is now moving away from perovskite materials such as $\mathrm{BaTiO}_{3}$ and PZT and growing to cover a wider spectrum of ferroelectric materials. New areas of study include polymeric ferroelectric systems such as poly(vinylidene fluoride) [120] and more widely $\mathrm{LiNbO}_{3}$. What is particularly interesting in the case of $\mathrm{LiNbO}_{3}$ is the variation in the details about the published results. When the work relating to perovskite materials is reviewed there are no publications that demonstrate homogenous wire like structures that are being formed on the ferroelectric surface. In all cases the material that formed on the surface has done so from discrete nucleation sites. The reasons for this are that in materials such as PZT and $\mathrm{BaTiO}_{3}$ the spontaneous polarisation is among the largest for ferroelectric materials.

Certainly when compared to materials such as $\mathrm{LiNbO}_{3}$ there are significant, an order of magnitude or greater, differences in the spontaneous polarisation. So what does this mean? In essence for the materials with a large spontaneous polarisation the inner Helmholtz layer is extremely tightly bound to the surface. This means that in order for local REDOX chemistry to occur there must be a local disruption, as noted earlier, in this layer. Therefore it is on regions of defect that the initial nucleation takes place [92]. For a material, such as $\mathrm{LiNbO}_{3}$, the inner layer is not as tightly bound as the spontaneous field is lower. This then means that it is possible for there to be a more homogeneous nucleation of growing material on the surface of the ferroelectric. There is likely to be more solvated ions (cations for reduction over a positive domain) close to the surface than in the case of a strongly bound layer. The early discussion over the use of $\mathrm{LiNbO}_{3}$ showed a variety of growth mechanisms which when considered in the light of spontaneous polarisation and power of incident radiation can be found to be mutually supportive [66, 121-123]. A further anomaly of $\mathrm{LiNbO}_{3}$ is the low electron affinity

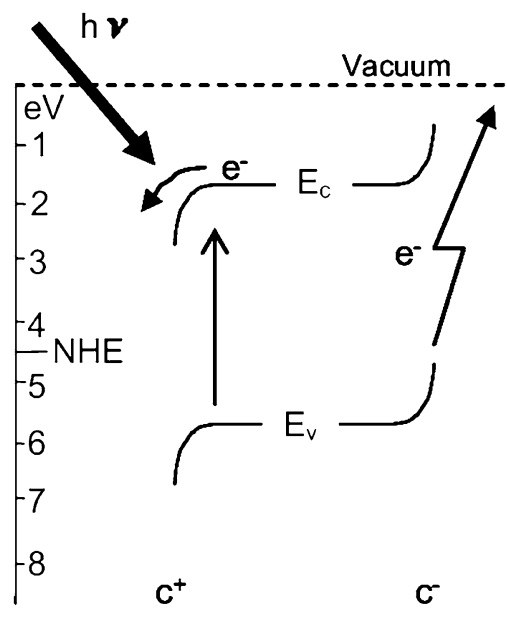

Fig. 14 Band diagram for $\mathrm{LiNbO}_{3}$ showing location of the conduction and valence bands with respect to vacuum and the standard electrochemical series. Electrons can be promoted on $\mathrm{c}^{-}$to react with ions in solution through a photoelectric effect and on the $\mathrm{c}^{+}$domain through ferroelectric depolarisation effects, as seen on other systems (with permission from Ref. 123)

found for $\mathrm{LiNbO}_{3}$, as shown in Fig. 14. This means that when high energy photons are used to irradiate the surface it is possible the produce electrons from both the $\mathrm{c}^{+}$and $\mathrm{c}^{-}$ domain surfaces. The low spontaneous polarisation and low electron affinity combine to make some extremely effects on the material.

\section{Conclusions}

Although ferroelectric conduction can be modelled as wide bandgap semi-conduction from the perspective of generalised charge concentrations and mobilities, it should be noted that influences of the spontaneous polarisation of a ferroelectric function to either counteract or enhance different aspects of the material's properties. Whilst the bound structural charge produces strong depolarising fields across grains, the free charge gradients are enhanced across the bulk in line with domain polarities.

Surface reactivity is reliant upon properties inherent in the whole system and so the overall effects of charge gradients at boundaries, interfaces and therefore ultimately on reaction surfaces must be considered.

In this review we also focussed on the new developments of patterned surfaces being used for selected chemical reactions and possible future implications. The state of the art approach to the directed assembly of complex nanostructures (oxide substrates, metal nanoparticles and organic and biological molecules) has been discussed which opens new avenues to nanodevice integration. 
Open Access This article is distributed under the terms of the Creative Commons Attribution Noncommercial License which permits any noncommercial use, distribution, and reproduction in any medium, provided the original author(s) and source are credited.

\section{References}

1. Kalinin SV, Bonnell DA, Alvarez T, Lei X, Ferris JH, Dunn S, Zhang Q (2002) Nanoletters 2:589

2. Lines ME, Glass AM (eds) (1977) Principles and applications of ferroelectric and related materials. Clarendon Press, Oxford

3. Hench LL, West JK (eds) (1990) Principles of electronic ceramics. Wiley, New York

4. Setter N, Colla EL (eds) (1993) Ferroelectric ceramics. Birkhauser Verlag, Basel

5. Alexe M, Hesse D (2006) J Mater Sci 41:1. doi:10.1007/ s10853-005-5192-x

6. Cross LE (2006) J Mater Sci 41:53

7. Valasek J (1920) Phys Rev 15:537

8. Polla DL, Francis LF (1998) Annu Rev Mater Sci 28:563

9. Dabbs DM, Aksay IA (2000) Annu Rev Phys Chem 51:601

10. Schonholzer UP, Gauckler LJ (1999) Adv Mater 11:630

11. Doodeve CF, Kitchener JA (1938) Trans Farrady Soc 34:902

12. Mashio F et al (1956) Abstract book of annual meeting chemical society of Japan, p 223

13. Fujishima H, Honda K (1972) Nature 238:37

14. Nasby RD, Quinn RK (1976) Mater Res Bull 11:985

15. Gioncondi GL, Rohrer GS (2001) Chem Mater 13:241

16. Fridkin VM (1980) Ferroelectric semiconductors. Plenum Publishing, NY, ISBN 0-306-10957-3

17. Koops HWP, Dobisz E (1997) J Urban Scale Sci Technol B15:1369

18. Xia Y, Whitesides GM (1998) Ann Rev Mater Sci 28:153

19. Chou SY (2001) MRS Bull 26:512

20. Loweth CJ, Caldwell WB, Peng X et al (1999) Angew Chem Int Ed 38:1808

21. Park S, Lazarides AA, Mirkin CA, Letsinger RL (2001) Angew Chem Int Ed Engl 40:2909

22. Whaley SR, English DS, Hu EL, Barbara PF, Belcher AM (2000) Nature 405:665

23. Piner RD, Zhu J, Xu F, Hong S, Mirkin CA (1999) Science 283:661

24. Hughes TR et al (2001) Nat Biotechnol 19:342

25. Duan X, Huang Y, Cui Y, Wang J (2001) Nature 409:66

26. Dunn S, Cullen D, Abad-Garcia E, Bertoni C, Carter R, Howorth D, Whatmore RW (2004) App Phys Lett 85:3537

27. Zhao MH, Bonnell DA, Vohs JM (2008) Surf Sci 602:2849

28. Li DB, Zhao MH, Garra J, Kolpak A, Rappe AM, Bonnell DA, Vohs JM (2008) Nat Mater 7:473

29. Habicht S, Nemanich RJ, Gruverman A (2008) Nanotechnology 19:495303

30. Kalinin SV, Bonnell DA (2002) Phys Rev B 65:125408

31. Dunn S, Shaw CP, Huang Z, Whatmore RW (2002) Nanotechnology 13:456

32. Li DB, Strachan DR, Ferris JH, Bonnell DA (2006) J Mater Res 21(4):935

33. Jona F, Shirane G (1993) Ferroelectric crystals. Dover Publications, New York

34. Jones PM, Dunn S (2009) J Phys D Appl Phys 42:065408

35. Dunn S, Jones PM, Gallardo DE (2007) J Am Chem Soc 129:8724

36. Jones PM, Gallardo DE, Dunn S (2008) Chem Mater 20:5901

37. Lei X, Li D, Shao R, Bonnell DA (2005) J Mater Res 20(3):712
38. Eng LM (1999) Nanotechnology 10:405

39. Emelyanov AY, Pertsev NA, Salje EKH (2001) J Appl Phys 89:1355

40. Xiao Y, Shenoy VB, Bhattacharya K (2005) Phys Rev Lett 95:247603

41. Smolenskii GA, Bokov VA, Isupov VA, Krainik NN, Pasynkov RE, Sokolov AI (1984) Ferroelectrics and related materials. Gordon and Breach, NewYork

42. Jaffe B, Cook WR Jr, Jaffe H (1971) Piezoelectric ceramics. Academic Press, London

43. Bonnell DA (1998) Prog Surf Sci 57:187

44. Wang YG, Dec J, Kleemann W (1998) Appl Phys 84:6795

45. Gruverman AL, Hatano J, Tokumoto H (1997) Jpn J Appl Phys A $36: 2207$

46. Takashige M, Hamazaki SI, Shimizu F, Kojima S (2000) Ferroelectrics $240: 1359$

47. Pang GKH, Baba-Kishi KZ (1998) J Phys D 31:2846

48. Eng LM, Friedrich M, Fousek J, Günter P (1996) J Vac Sci Technol B 14:1191

49. Correia A, Massanell J, Garcia N, Levanyuk AP, Zlatkin A, Przeslawski J (1996) Appl Phys Lett 68:2796

50. Saurenbach F, Terris BD (1990) Appl Phys Lett 56:1703

51. Devonshire AF (1954) Adv Phys 3:85

52. Boerasu I, Pereira M, Gomes MJM, Ferreira MIC (2000) J Optoelectron Adv Mater 2:602

53. Boerasu I, Pereira M, Gomes MJM, Vasilevskiy MI, Costa MF (2002) Ferroelectrics 268:187

54. Souni ME, Piorra A, Solterbeck CH, Abed M (2001) Mater Sci Eng B 86:237

55. Scott JF (2000) Ferroelectric memories. Springer, New York

56. Yarmarkin VK, Gol'tsman BM, Kazanin MM, Lemanov VV (2000) Phys Solid State 42:511

57. Mihara T, Watanabe H, Yoshimori H (1992) Integr ferroelectrics 1:269

58. Riege H, Boscolo I, Handerek J, Herleb U (1998) J Appl Phys $84: 1602$

59. Shur D, Rosenman G (1996) J Appl Phys 80:3445

60. Yang WC, Rodriguez BJ, Gruverman A, Nemanich RJ (2005) J Phys Condens Matter 17:S1415

61. Kalinin SV, Bonnell DA, Alvarez T, Lei X, Hu Z, Shao R, Ferris JH (2004) Adv Mater 16:795

62. Kanzig W (1955) Phys Rev 98:549

63. Merz WJ (1956) J Appl Phys 27:938

64. Treibwaser S (1960) Phys Rev 118:100

65. Pintilie L, Boerasu I, Gomes M, Zhao T, Ramesh R, Alexe M (2005) J Appl Phys 98:124104

66. Hanson JN, Rodriguez BJ, Nemanich RJ, Gruverman A (2006) Nanotechnology 17:4946

67. Tiwari D, Dunn S (2009) J Eur Ceram Soc. doi:10.1016/ j.jeurceramsoc.2009.03.18

68. Tiwari D, Dunn S, Zhang Q (2009) Mater Res Bull (Online)

69. Scott JF (1999) Aust J Phys 52:903

70. Gratzel M (2001) Nature 414:338

71. Ambacher O, Smart J, Shealy JR, Weimann NG, Chu K, Murphy M, Dimitrov R, Wittmer L, Rieger W, Hilsenbeck J (1999) J Appl Phys 85:3222

72. Kalinin SV, Bonnell D (2001) Phys Rev B 63:125411

73. Tybell T, Ahn CH, Antognazza L, Trisconne JM (1998) Vide Sci Tech Appl 289:551

74. Dunn S, Tiwari D, Jones P, Gallardo D (2007) J Mater Chem $17: 4460$

75. Kalinin SV, Shao R, Bonnell DA (2005) J Am Ceram Soc 88(5): 1077

76. Kalinin SV, Bonnell DA (2004) Nano Lett 4:555

77. Greuter F, Blatter G (1990) Semicond Sci Technol 5:111

78. Gupta TK (1990) J Am Ceram Soc 73(7):1817 
79. Lee HY, Villamil SS, Burton LC (1986) In: Proceedings of the 6th international symposium on applications of ferroelectrics, pp 361-366

80. Villamil SS, Lee HY, Burton LC (1987) IEEE Trans Comput Hybrids Manuf Technol HMT 12(4):482

81. Neumann H, Arlt G (1986) Ferroelectrics 69:179

82. Chiang YM, Tagaki TJ (1990) J Am Ceram Soc 73(11):3278

83. Van de Krol R, Tuller HL (2002) Solid State Ionics 150:167

84. Blatter G, Greuter F (1986) Phys Rev B 34:8555

85. Jones PM, Dunn S (2007) Nanotechnology 18:185702

86. Maier J (1993) Angew Chem Int Ed 32:313

87. Noland TA (1964) Phys Rev 134:A511

88. Franklin AD (1972) In: Crawford JH Jr, Slifkin LM (eds) Point defects in solids, vol 1. General and ionic solids. Plenum Press, New York, $p 1$

89. Brody PS, Crowne F (1975) J Electron Mater 4(5):955

90. Pleskov YV, Gurevich YY (1986) Semiconductor photoelectrochemistry. Consultants Bureau, Plenum Publishing Corporation, New York

91. Singh J (1995) Semiconductor optoelectronics. Electrical Engineering Series, McGraw-Hill International Editions, McGrawHill Inc., NY

92. Dunn S, Sharp S, Burges S (2009) Nanotechnology 20:115604

93. Bard AJ, Faulkner LR (1980) Electrochemical methods. Wiley, NY

94. Scott JF (2000) In: Itoh K, Sakurai T (eds) Advanced microelectronics. Springer, Berlin

95. Gerischer H (1970) Physical chemistry, an advanced treatise, vol 1XA, Chap 5. Academic Press, New York

96. Minoura H, Oki T, Tsuiki M (1976) Chem Lett 1279

97. Boddy PJ (1969) Surf Sci 13:52

98. Tomkiewicz M, Fay H (1979) Appl Phys A 18:1

99. Brady JJ, Moore WH (1939) Phys Rev 55:308

100. Fridkin VM (1979) Photoferroelectrics, Chap 6. Springer, Berlin

101. Chynoweth AG (1956) Phys Rev 102:705
102. Chen FS (1967) J Appl Phys 40:3389

103. Brody PS (1973) Solid State Commun 12:673

104. Cox GA, Roberts GG, Tregold RH (1966) J Appl Phys 17:743

105. Brody PS, Vrabel MJ (1968) Bull Am Phys Soc 13:617

106. Glass AM, von Linde D, Megran TJ (1974) J Appl Phys Lett 25:233

107. Brody PS (1973) Bull Am Phys Soc 18:65

108. Brody PS (1973) Bull Am Ceram Soc 52:631

109. Brody PS (1975) J Solid State Chem 12:193

110. Inoue Y, Sato K, Sato K (1989) J Chem Soc Faraday Trans 1 85(7): 1765

111. Pensak L (1958) Phys Rev 109:601

112. Merz W (1958) Helv Phys Acta 31:625

113. Wrighton MS, Morse DL, Ellis AB, Ginley DS, Abrahamson HB (1976) J Am Chem Soc 98:44

114. Butler MA, Nasby RD, Quinn RK (1976) Solid State Commun 19:1011

115. Mavroides JG, Tchernev DI, Kafalas JA, Kolesar DF (1975) Mater Res Bull 10:1023

116. Wrighton MS, Ellis AB, Wolczanski PT, Morse DL, Abrahamson HB, Ginley DS (1976) J Am Chem Soc 98:2774

117. Okuda M, Yoshida K, Tanaka N (1976) Jpn J Appl Phys 15(8):1599

118. Inoue Y, Okamura M, Sato K (1985) J Phys Chem 8:5184

119. Inoue Y, Sato K, Sato K, Miyama H (1986) Chem Phys Lett 129:79

120. Rankin C, Chou CH, Conklin D, Bonnell DA (2007) ACS Nano $1: 234$

121. Yang WC, Rodriguez BJ, Gruverman A, Nemanich RJ (2004) Appl Phys Lett 85:2316

122. Liu X, Kitamura K, Terabe K, Hatano H, Ohashi N (2007) Appl Phys Lett 1:044101

123. Dunn S, Tiwari D (2008) Appl Phys Lett 93:092905

124. Abe R, Sayama K, Sugihara H (2005) J Phys B Chem 109:1606 